\title{
Preparation of File (Sohan) by using the Probiotics with Chemical and Microbiologic Analysis for Individual's Health
}

\section{Neda Mostowfi ${ }^{*}$, Sedigheh Mehrabian² and Masoomeh Mirzai}

${ }^{1}$ School of Life Sciences, Islamic Azad University, North Tehran Branch, Iran

${ }^{2}$ Department of Microbiology, Faculty of Sciences, Islamic Azad University, North Tehran Branch, Iran

\begin{abstract}
Background: Now-a-days, because of the tendency of consumers to Probiotics products, production and consumption of these products have been a growing trend. Sohan due to the presence of useful ingredients such as wheat germ, honey, saffron, etc. is full of nutrients; but high levels of cholesterol and sugar in Sohan cause limited consumption especially in the case of people with diabetes and high cholesterol levels.
\end{abstract}

Objectives: The aim of this study is an investigation of probiotics beneficial effects on the Sohan (traditional Persian saffron brittle toffee). So, in this study, our target is preparing of a Sohan with low in fat and sugar.

Material and methods: Two microorganisms including Lactobacillus acidophilus and Bacillus coagulans, were used in this study. Bile tolerance test, motility test, heat resistance and antibiotic tests were performed to confirm the probiotic microorganisms. After that, these microorganisms were added to the Sohan. Then, these microorganisms were added to the Sohan. After three repetitions of tests at different times, before and after the addition of probiotics, parameters such as glucose, cholesterol, triglycerides, and the number of microorganisms were measured by different methods.

Results: The results showed that the number of microorganisms in time periods of test from the time of inoculation to one month has been downward. PH value in all the samples remained almost constant. The amount of glucose, triglyceride and cholesterol were declined significantly in the Sohan containing the Lactobacillus acidophilus. However, in samples containing Bacillus coagulans, decrease of the triglyceride level and increase of the cholesterol and glucose levels was observed.

Conclusion: According to the results, Sohan containing probiotics can be an appropriate alternative for consumption by people with the special diet.

\section{Keywords: Sohan; Microorganisms; Probiotics; Health}

\section{Introduction}

Sohan is one of the Iranian traditional foods and due to the beneficial ingredients, such as wheat germ, honey, and saffron, (etc.) is full of nutrients. However, high levels of cholesterol and sugar in Sohan have limited its consumption especially in the case of people with diabetes and high level of cholesterol. Chemical ingredients of Sohan are sugar, honey and Badka as a full of nutrients carbon hydrates that sugar and Badka contain cellulose and glucose respectively. Also, honey contains about $80 \%$ carbohydrates and its remnants are protein, minerals, aromatic elements, enzymes, vitamins, pollen and a little water. Butter is source of cholesterol as an animal lipid. White flour is a highly nutritious ingredient and contains vitamins B1, B2, E, fat and starch ingredients, gluten, sucrose, lecithin, variety of pentose, nucleic acids, phytin and amylase, phytase, protease, lipase, maltase, tyrosinase, peroxidase and oxalic acid enzymes. Wheat germ flour also has above ingredients but in the beginning of germination process the starch in the bud was broken and converted to digestible simple sugars. Proteins are converted to amino acid and oils and lipids also are converted to lipid acid by lipase enzyme in the bud. Rosewater, cardamom and saffron were used as natural colors and flavors.

Probiotics are defined as live microorganisms that are caused to health in the host body by sufficient quantities [1-3]. In the past Probiotics were used as nutritional supplements in the animal feed; however, today is also used for human host. Probiotics help to the treatment of kidney stones, preventing from constipation in the elderly, absorption and production of vitamin, digestion of lactose in the colon and reducing blood cholesterol and carcinogens and preventing from allergy etc. [4,5].
Probiotics have balanced the microflora of gastrointestinal system and preserve epithelial cells function, immune activity of mucous layer [6] and increase IgA directly [7]. Much of the probiotics belong to the lactic bacteria species (Lactobacilli) and hence they also are said lantibiotics [8]. Traditionally, cold dairy products are the preferred method for the production and cultivation of probiotics [9]. But recently researches have shown that productive bacteria of lactic acid particularly Bacillus remained stable in the baked foods and maintain the probiotics benefits in the baking temperature. These bacteria can survive in the high temperature because of production of spores. Bacillus coagulans is one of the Bacillus that can be used in the baking process due to the intrinsic properties [10].

The aim of this study is producing a Sohan with less level of sugar and lipid. According to the importance of the use of probiotics in the food industry, another purpose of the present paper is chemical analysis of probiotics in order to measure glucose, $\mathrm{pH}$, cholesterol and triglycerides and investigation of the growth and survival of probiotics in the nutritional ingredient. Providing healthy and beneficial food can be considered one of functional objectives of this study if a positive

*Corresponding author: Neda Mostowfi, School of Life Sciences, Islamic Azad University, North Tehran Branch, Iran, Tel: +98-021-22716582; E-mail: N.mstofi@gmail.com

Received May 19, 2017; Accepted June 08, 2017; Published June 15, 2017

Citation: Mostowfi N, Mehrabian S, Mirzai M (2017) Preparation of File (Sohan) by using the Probiotics with Chemical and Microbiologic Analysis for Individual's Health. J Food Process Technol 8: 679. doi: 10.4172/2157-7110.1000679

Copyright: ( $) 2017$ Mostowfi N, et al. This is an open-access article distributed under the terms of the Creative Commons Attribution License, which permits unrestricted use, distribution, and reproduction in any medium, provided the original author and source are credited. 
result obtain from this investigation and color, smell and taste of Sohan does not change.

\section{Materials and Methods}

\section{Bacterial cultivation}

Cultivation of Lactobacillus acidophilus was done in MRS culture medium at $37^{\circ} \mathrm{C}$ for 24 hours and the cultivation of Bacillus coagulans was done in the nutrient culture medium at $37^{\circ} \mathrm{C}$ for $24-48$ hours.

\section{Microbial and biochemical tests}

Morphological investigation, Gram stain and biochemical tests such as catalase, motion, the ability to ferment of glucose, fructose and galactose sugars were carried out to prove the integrity and purity of the colonies.

\section{Affirmation of probiotic bacteria}

In order to prove the investigation of probiotic, most work has been done in laboratory conditions was creation of internal conditions of gastrointestinal system, using various $\mathrm{PH}$ and different concentrations of bile salts at varying times to determine the survival of the under-test species $[11,12]$.

\section{Salt tolerance test}

Tests was done in a dilution of $0.5,2.5,5,10$ and $20 \% \mathrm{NaCl}$ with first number of McFarland and tubes were placed in the incubated for 24 hours at $37^{\circ} \mathrm{C}$. Turbidity of pipe created by bacteria growth in the culture medium reported as $+2,+1$ and - (minus) and power-plate was done in the MRS Agar and Mueller Hinton Agar culture medium [11-16].

\section{Fermentation of sugars test}

Sugar compounds were used to create MRS culture medium but instead of glucose and beef extract, $2 \%$ arabinose, galactose, fructose, mannitol, sucrose, sorbitol, trehalose, raffinose, xylose, rhamnose. The maltose sugars added separately to Lactobacillus and the galactose, fructose, lactose, trehalose, sucrose, Slbiose and maltose sugars were added to the yeast and microorganisms are cultivated in them. The results after 24, 48 and 72 hours was recorded [17-25].

\section{Heat resistance test}

This test was done in this way that fresh cultivation of the sample prepared and after 24 hours incubation equivalent to half McFarland of each sample was fused in the physiology serum and then the number of bacteria was recorded by the spectrophotometer device. The samples were putted for 20 minutes at temperatures of 80,60 and 40 bain marie and the bacteria were counted after 20 minutes at different temperatures by device. However, after putting samples at different temperatures of bain marie for 20 minutes, pure-plate was prepared of each of the pipes as serial and was putted for 24 hours at $37^{\circ} \mathrm{C}$ incubator and the stability and resistance of the samples was determined [25].

\section{Bile tolerance test}

At first $0.5 \mathrm{McF}$ arland was suspension in each $\mathrm{ml}$ of isotonic buffer containing 0.2 pepsin with $\mathrm{PH} 2$ and also 7 (as a control) and was putted at incubator to determine the resistance to acid and pepsin. Then, their stability using pure-plate method and light absorption of samples were measured at wavelength of $560 \mathrm{~nm}$ at the time of $0,1,2$ and 3 hours.

\section{Evaluation of motion test}

SIM semi-solid culture medium was used for motion test. After preparing the SIM culture medium according to the instruction of its manufacturer factory, microorganisms were cultured in the SIM tube and the results were evaluated after 24 hours incubation at $37^{\circ} \mathrm{C}$.

\section{Resistance to antibiotics}

At first Muller Hinton agar culture medium was created to evaluate antibiotic resistance of samples. Then half McFarland solution was prepared from Lactobacillus and Bacillus coagulans samples and culture was prepared by swab on Mueller Hinton agar culture medium. Then antibiotic discs were putted on the culture medium. The halo of lack of growth created around antibiotic discs was measured after 24 hours incubation at $37^{\circ} \mathrm{C}[21]$.

\section{Proving the microorganism's resistance in cooked Sohan}

At first Sohan was prepared and equivalent to half McFarland bacteria added to them and CFU was measured at time of zero. Then Sohan was putted at room temperature and $10 \%$ solution of the samples was prepared at different times $(24,48,72,168$, and...) and pure-plate was provided and incubated for 24 hours at 37 degrees and the CFU was calculated and recorded [25].

\section{Glucose, cholesterol and triglycerides test}

Special kits were used to measure any parameters of glucose, cholesterol and triglycerides in the prepared Sohan and then after adding microorganisms measured at the times of zero, 24,48,72, a week, a month and were compared with the initial value $[17,18]$.

\section{Results}

\section{Biochemical tests}

Bacillus coagulans: Results of tests showed that Bacillus coagulans is Gram-positive, spore-bearing, animated and positive Kalataz and positive oxidase. Sugar fermentation test also showed that this bacterial can ferment 5 tested sugars and since these findings were consistent with the specifications of Bacillus coagulans, it is proved that the intended bacterium is same Bacillus coagulans.

Lactobacillus: This is microorganism of gram-positive, sporefree, sedentary bacilli and negative catalase and oxidase. According to the results of the sugars fermentation and heat resistance tests, it was proved that is the same intended Lactobacillus (Table 1).

Sensitivity to antibiotics test: Results of antibiotic sensitivity investigation suggested that Lactobacillus acidophilus compared to cephalotin, nalidyik acid, gentamicin, imipenem, lincomycin had been resistant but compared to tetracycline, amoxicillin, clindamycin, nitroforanshin trimethoprim and sulfamethoxazole was sensitive (Table 2). Also, Bacillus coagulans compared to amoxicillin and ancomycin had been resistant but compared to tetracycline, gentamicin, ampicillin, chloramphenicol, sefamandel, penicillin, neomycin, cephalexin, trimethoprim and sulmaksazol had been sensitive (Table 3).

Fermentation of sugars test: Lactobacillus acidophilus could ferment mannitol, galactose, trehalose and raffinose sugars and it was changed ambient color from red to yellow; but it couldn't ferment sorbitol, glucose, lactose, fructose, sucrose, xylose, rhamnose and arabinose and ambient color hadn't been changed. Bacillus coagulans bacteria could ferment glucose, mannose, lactose, galactose, fructose and arabinose and it changed the ambient color from yellow to red.

Glucose, cholesterol and triglyceride test: After doing the cholesterol test using cholesterol measurement kit and turbidity measurement test by spectrophotometer, it was shown that cholesterol 
Citation: Mostowfi N, Mehrabian S, Mirzai M (2017) Preparation of File (Sohan) by using the Probiotics with Chemical and Microbiologic Analysis for Individual's Health. J Food Process Technol 8: 679. doi: 10.4172/2157-7110.1000679

Page 3 of 5

\begin{tabular}{|c|c|c|c|c|}
\hline Biochemical tests & Gram stain & Catalase test & Motion test & TSI \\
\hline Probiotic microorganisms & Gram-negative bacilli & - & - \\
\hline Lactobacillus acidophilus & Oram-positive bacilli string & + & Yellow color & + \\
\hline Bacillus coagulans & Yellow color & + \\
\hline
\end{tabular}

Table 1: Biochemical test results.

\begin{tabular}{|c|c|c|c|c|c|c|c|c|c|c|}
\hline Antibiotic Disk & TE & AMX & CC & $\mathbf{L}$ & SXT & TPM & NA & CF & GM & V \\
\hline Lactobacillus acidophilus & $\begin{array}{c}s \\
26\end{array}$ & 24 & $\begin{array}{l}\mathrm{R} \\
0\end{array}$ & $\begin{array}{l}r \\
0\end{array}$ & $\begin{array}{c}\mathrm{s} \\
36\end{array}$ & $\begin{array}{c}\mathrm{i} \\
11\end{array}$ & $\begin{array}{l}r \\
0\end{array}$ & $\begin{array}{l}r \\
0\end{array}$ & $\begin{array}{l}\mathrm{i} \\
9\end{array}$ & $\begin{array}{r}S \\
24\end{array}$ \\
\hline
\end{tabular}

Table 2: Sensitivity Lactobacillus acidophilus antibiotic based on the inhibition zone diameter (in $\mathrm{mm}$ ).

\begin{tabular}{|c|c|c|c|c|c|c|c|c|c|c|c|c|}
\hline Antibiotic Disk & TE & AMX & $\mathbf{P}$ & $\mathbf{L}$ & C & AM & $\mathbf{N}$ & $\mathrm{CN}$ & GM & $\mathrm{CM}$ & SXT & v \\
\hline Bacillus coagulans & 30 & 9 & 21 & 17 & 23 & 29 & 24 & 30 & 34 & 28 & 30 & 17 \\
\hline
\end{tabular}

Table 3: Sensitivity Bacillus coagulans antibiotic based on the inhibition zone diameter (in $\mathrm{mm}$ ).

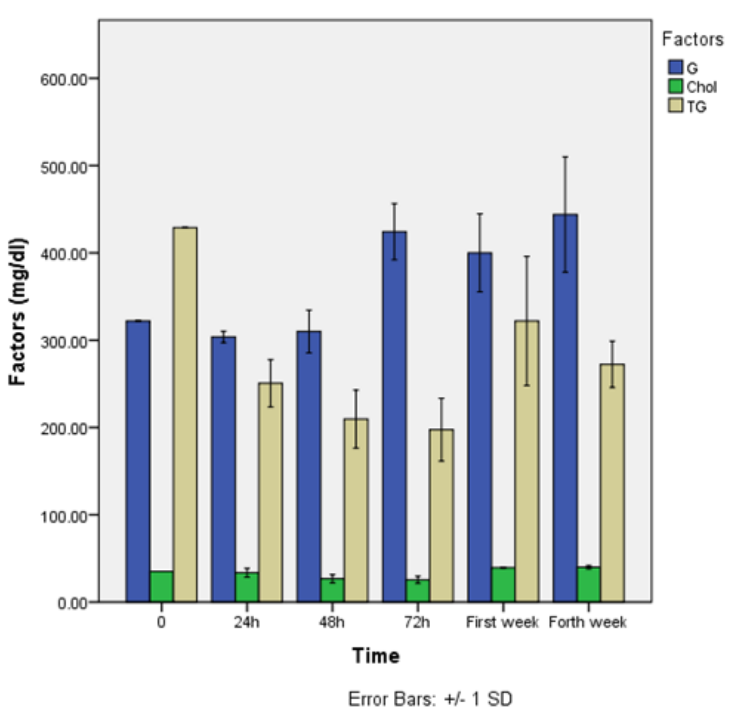

Figure 1: Changes in glucose, triglycerides and cholesterol by Bacillus coagulans.

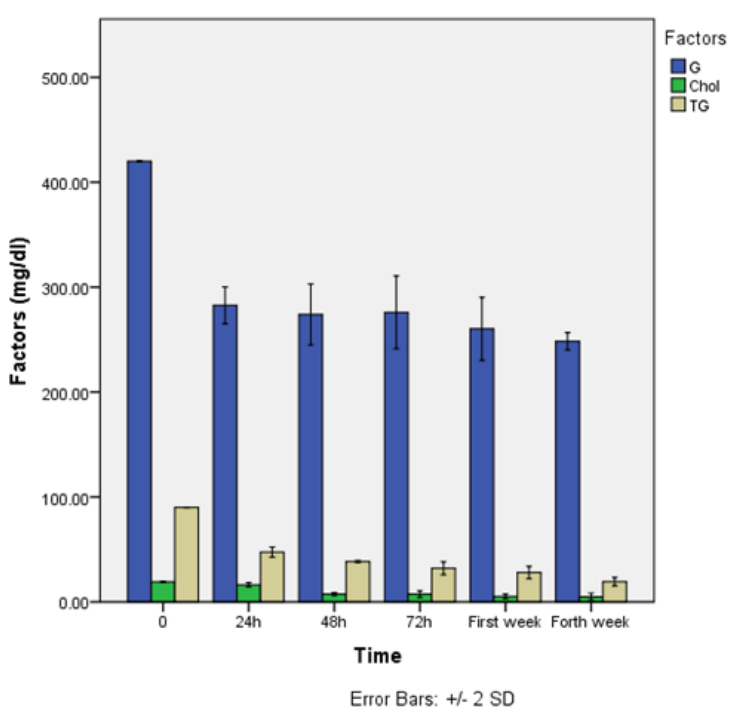

Figure 2: Changes in glucose, triglycerides and cholesterol by Lactobacillus acidophilus.

level in both samples of Sohan have been declined dramatically because of the growth of microorganisms. After doing the triglyceride test using triglyceride measurement kit and turbidity measurement test by spectrophotometer, it was shown that triglyceride level has been dramatically changed in both samples of Sohan.

At first glucose levels were measured in three samples of Sohan using the Fehling reagent that did not show an accurate result. Then, using a glucose measurement device has been shown that Lactobacilli reduce glucose level of Sohan and Bacillus coagulans increased glucose levels of Sohan. In other words, Lactobacilli have used glucose of Sohan and reduced while Bacillus coagulans converted sucrose into glucose and glucose level has increased (Figures 1 and 2).

Maintenance of microorganisms in Sohan: To investigate survival of microorganisms after adding microorganisms to Sohan in times of 24 hours, 48 hours, 72 hours, one week and four weeks, at first $1 \mathrm{~g}$ of each sample of Sohan was fluxed in $10 \mathrm{ml}$ of water and was prepared a dilution series. Then each tube was transferred to the BHI agar culture medium. After 24 hours, the number of colonies was counted and multiplied by the intended dilution. The results showed that Lactobacillus acidophilus had been a descending growth process and disappeared completely in less than a month; but Bacillus coagulans had been less descending growth process and had remained more than a month in the Sohan that was satisfactory.

Sensory evaluation: Results of taste test show that probiotics Sohan in cases of odor, flavor and texture isn't distinctly different from typical one and even in opinion of majority of persons probiotic Sohan made by Bacillus coagulans was more palatable than the typical one; that this is a good result for this research (Table 4).

\section{Discussion}

Sohan and confectionery products are popular in Iran. Adding probiotics can strengthen positive and beneficial aspects of these foods. Due to the beneficial effects of probiotics, in addition to their beneficial changes in the Sohan, conversion of sugar into glucose and fructose which is the preparation of invert sugar and change in cholesterol and triglycerides level and survival of probiotics in the Sohan that most people desire to consume this type of nutritional ingredient. Probiotics are useful for the health and preventing from detrimental effects of additives. Tested microorganisms in this study are consistent with measures expressed about probiotics in the Hand book of probiotic. These bacteria with low $\mathrm{pH}$ can resist in the first step that enter to stomach and survive and are resistant to bile and pepsin as well. In this study, it is tried to provide conditions of gastrointestinal system such as different values of $\mathrm{pH}$, creating varying concentrations of bile salts at varying times to determine the survival time of under test species. 
Citation: Mostowfi N, Mehrabian S, Mirzai M (2017) Preparation of File (Sohan) by using the Probiotics with Chemical and Microbiologic Analysis for Individual's Health. J Food Process Technol 8: 679. doi: 10.4172/2157-7110.1000679

\begin{tabular}{|c|c|c|c|c|}
\hline Sohan Features & \multicolumn{2}{|c|}{ Control } & \multicolumn{2}{c|}{ Coagulans probiotic } \\
\hline Privilege & Percent & Average & Percent & Average \\
\hline Color & 100 & 5 & 100 & 5 \\
\hline Smell, Taste, color & 95 & 4.75 & 100 & 5 \\
\hline Chewiness & 90 & 4.5 & 100 & 5 \\
\hline Sohan tissue & 90 & 4.5 & 100 & 5 \\
\hline Rasp grading & 100 & 5 & 100 & 5 \\
\hline
\end{tabular}

Table 4: The results of the taste test poll.

This study is consistent with the features listed in World journal of dairy food science that mentioned best selection criteria for probiotics.

According to the conducted researches by Oryan and Yaghmaei [24], it was observed that in yoghurts prepared with different ratios of Lactobacillus acidophilus, the number of bacteria reached $10^{8}$ after incubation times and almost the same number was maintained during incubation time that this shows the stability of probiotics in yogurt. The results obtained from this study indicate the instability of Lactobacillus acidophilus in Sohan and therefore the use of this bacterium is not desirable to prepare a probiotic [25]. Bacillus coagulans was also counted that showed Bacillus coagulans is more resistant and they reduce less after one month.

Optimum PH for bacteria in the probiotic yogurt is 4.2-4.6 which $\mathrm{PH}$ difference of probiotics yogurts in intended times has compared to each other in the calculation of $\mathrm{PH}$ changes. However, there wasn't a significant difference in $\mathrm{PH}$ changes during different days that indicated that $\mathrm{PH}$ is stable during incubation of product. This matter (stability of $\mathrm{PH}$ ) like the stability of used bacteria is one of the important issues in preparing a probiotic product because decrease of $\mathrm{PH}$ during incubation of product to low values $(\mathrm{PH}<3)$ is associated with increase of acid produced by bacteria that affected the taste of products and create unfavorable conditions for the product [25]. In this study, after fusion of microorganisms to Sohan, $\mathrm{PH}$ of Sohan was measured at different intervals that there was no significant change. Results of this study are consistent with the findings of Oryan and Yaghmaei [24]. Clinical studies show that Lactobacillus cells in in vitro conditions can decrease added cholesterol to the culture medium and in in vivo conditions also can reduce serum cholesterol. Jenkins [15] showed that increase of lipid in culture medium reduces the synthesis of lipid acids by lactic acid bacteria [20]. The findings indicate that probiotics are capable of absorbing cholesterol into their cell membranes in the presence of bile [23].

In this study, after adding Lactobacillus acidophilus to Sohan, cholesterol and triglycerides level were measured by cholesterol and triglyceride measurement kit that Indicate a decrease of both variable and obtained results are consistent with above studies. But adding Bacillus coagulans to Sohan increased cholesterol level and triglyceride levels had decreased less. Using sugar in the preparation of Sohan increases level of cholesterol because sugar converts to glucose by bacteria and glucose plays an important role in the synthesis of cholesterol so it is recommended to use less sugar and glucose in the preparation of Sohan because it controls cholesterol and is also more affordable economically. Khatri et al. [13] conducted a study on preparing probiotic bread and results showed that Bacillus coagulans breaks down starch in the bread and increases the sugar after baking bread. In this study, the amount of sugar in control Sohan and probiotic Sohan and were measured using a glucose measurement device and compared. Bacillus coagulans increases sugar which shows the breakdown of sucrose and its conversion into glucose. This change was perceptible in the taste test.

In this study, our selected nutritional ingredient (Sohan) is covered by protective. It is recommended that organisms added to Sohan after baking Sohan and before cooling completely and stirred and finally shaped Sohan as desirable. Sohan must be covered with protective to provide better stability conditions for probiotics. Studies have also shown that probiotics have little stability during preparation for sale. Stability of probiotics can be increases by selecting specific types that is resistant to acid and bile, using impermeable containers to oxygen, cover, microencapsulation of bacteria with various polymers and adaptation to stress [19].

\section{Conclusion}

Finally, it can be concluded that probiotics Sohan due to its advantages such a useful microorganism, lower level of glucose, triglycerides and cholesterol than control Sohan is better options for diabetics, people with high cholesterol and even healthy people.

\section{References}

1. FAO/WHO (2001) Health and nutritional properties of probiotics in food including powder milk with live lactic acid bacteria, World Health Organization, Geneva, Switzerland.

2. Aldemita RR, Reaño IME, Solis RO, Hautea RA (2014) Trends in global approvals of biotech crops. GM Crop Food 6: 150-166.

3. De Vecchi E, Drago L (2006) Lactobacillus sporogenes or Bacillus coagolans Misidentification or mislabeling? Int J Probio Prebio 1: 3-10.

4. Le DH, Hong HA, Barbosa TM, Henriques AO, Cutting SM (2004) Characterization of bacillus probiotics available for human use. Appl Environ Microbiol 70: 2161-2171.

5. Maathuis AJH, Keller D, Farmer S (2009) Survival and metabolic activity of the Ganeden $\mathrm{BC}^{30}$ strain of Bacillus coagulans in a dynamic in vitro model of the stomach and small intestine. Benef Microbes 1: 31-36.

6. Baron MA (2009) Patented strain of Bacillus coagulans increased immune response to viral challenge. Postgrad Med 121: 114-118.

7. Douglas S, Alvarez P, Feldman S, Pezzullo JC, Krieger RD, et al. (2009) A prospective, randomized, double blind, placebo-controlled parallel-group dual site trial to evaluate the effects of a Bacillus coagulans-based product on functional intestinal gas symptoms. BMC Gastroenterol J Rheumatol 33 951-956.

8. Zadeh MF (2006) Antimicrobial biotechnology. Tehran University Press, Tehran, Iran.

9. Lenoir-Wijnkoop I, Jones PJ, Uauy R, Segal L, Milner J (2013) Nutrition economics food as an ally of public health. British J Nutri 109: 777-784.

10. Dolin BJ (2009) Effects of a proprietary Bacillus coagulans preparation symptoms of diarrhea a predominant irritable bowel syndrome. Method Find Exp Clin Pharmacol 31: 655-659.

11. Sudha R, Chauhan P, Dixit K (2010) Molecular typing and probiotic attributes of a new strain of Bacillus coagulans-Unique IS-2: A potential biotherapeutic agent. Genetic Eng Biotechnol J 8: 1-20.

12. Lee YK (2009) Hand book of probiotics \& probiotics. John Wiley \& Sons Inc Hoboken, New Jersey, USA.

13. Khatri I, Sharma S, Ramya TNC, Subramanian S (2016) Complete genomes of Bacillus coagulans s-lac and Bacillus subtilis TO-A JPC, two phylogenetically distinct probiotics. PLoS ONE 11: e0156745.

14. Palop A, Sala FJ, Condon S (1997) Occurrence of a highly heat-sensitive spore sub-population of Bacillus coagulans STCC 4522 and its conversion to a more heat-stable form. Appl Environ Microbiol 63: 2246-2251.

15. Jenkins SG, Schuetz AN (2012) Current concepts in laboratory testing to guide anti-microbial therapy. Mayo Clinic Proceeding 87: 290-308.

16. Viiard E, Bessmeltseva M, Simm J, Talve T, Aaspõllu A, et al. (2016) Diversity and stability of lactic acid bacteria in rye sourdoughs of four bakeries with different propagation parameters. PLoS ONE 11: e0148325.

17. Ashrafi F (2006) Microbiology practical. Ahsn, Tehran, Iran.

18. Thomas L (1998) Clinical laboratory diagnostics. TH-Books Verlagsgesllschaft, Frankfurt, Germany. 
Citation: Mostowfi N, Mehrabian S, Mirzai M (2017) Preparation of File (Sohan) by using the Probiotics with Chemical and Microbiologic Analysis for Individual's Health. J Food Process Technol 8: 679. doi: 10.4172/2157-7110.1000679

19. Chae CS, Kwon HK, Hwang JS, Kim JE, Im SH (2012) Prophylactic effect of probiotics on the development of experimental autoimmune Myasthenia Gravis. PLoS ONE 7: e52119.

20. Kumar M, Nagpal R, Kumar R, Hemalatha R, Verma V, et al. (2012) Cholesterol lowering probiotics as potential biotherapeutics for metabolic diseases. Experiment Diabet Res.

21. Sharma R, Kapila R, Dass G, Kapila S (2014) Improvement in Th1/Th2 immune homeostasis, antioxidative status and resistance to pathogenic $\mathrm{E}$. coli on consumption of probiotic Lactobacillus rhamnosus fermented milk in aging mice. Age 36: 9686.
22. Ooi LG, Liong MT (2010) Cholesterol-lowering effects of probiotics and prebiotics: A review of invivo and invitro findings. Int J Mol Sci 11: 2499-2522.

23. Yehia HM, Hassanein WA, Ibraheim SM (2015) Purification and characterization of the extracellular cholesterol oxidase enzyme from Enterococcus hirae. BMC Microbiol 15: 178.

24. Oryan S, Yaghmaei D (2009) Inoculated strains of Lactobacillus GG probiotic yoghurt production compared with regular yogurt and their impact on the reduction of lipid metabolites in the serum of male Wistar rats. Med J 16: 1-15.

25. Bakhshi Z, Bakhshy M (2009) Practical diagnostic bacteriology parsley. Jafari, Tehran, Iran. 